\title{
Maximizing production of cellulose nanocrystals and nanofibers from pre-extracted loblolly pine kraft pulp: a response surface approach
}

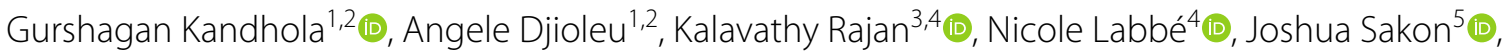
Danielle Julie Carrier ${ }^{3}$ (i) and Jin-Woo Kim ${ }^{1,2^{*}}$ (1)

\begin{abstract}
This study aims to optimize strong acid hydrolysis-based production of cellulose nanocrystals (CNCs) and cellulose nanofibers (CNFs) from pre-extracted and fully bleached kraft pulp of loblolly pinewood, the most abundant and commercially significant softwood species in southeastern United States. The effect of four parameters, including acid concentration, temperature, duration and pulp particle size, on the yield and properties of CNCs was investigated using the central composite design (CCD) of response surface methodology (RSM) for process optimization. While CNC yield was significantly affected by acid concentration and hydrolysis temperature and was adequately explained by an empirical model, none of the characteristic properties of CNCs, including crystallinity index, surface charge and particle size, displayed any strong correlation to the process parameters within the experimental ranges tested. At different hydrolysis severities, we not only analyzed the waste streams to determine the extent of holocellulose degradation, but also evaluated the properties of leftover partially hydrolyzed pulp, called cellulosic solid residues (CSR), to gauge its potential for CNF production via mechanical fibrillation. Conditions that maximized CNC yields $(60 \% \mathrm{w} / \mathrm{w})$ were $60 \%$ acid concentration, $58^{\circ} \mathrm{C}, 60$ min and 40 mesh particle size. Twenty percent $(\mathrm{w} / \mathrm{W})$ of the pulp was degraded under these conditions. On the other hand, conditions that maximized CSR yields ( $60 \% \mathrm{w} / \mathrm{w})$ were $54 \%$ acid, $45^{\circ} \mathrm{C}$, 90 min and 20 mesh particle size, which also produced $15 \%$ CNCs, caused minimal pulp degradation $(<5 \%)$ and imparted sufficient surface charge such that CSR was easily microfluidized into CNFs. Therefore, the strong acid hydrolysis process could be tuned to maximize the production of cellulose nanocrystals and nanofibers and obtain two products with different properties and applications through the process optimization.
\end{abstract}

Keywords: Loblolly pine, Kraft process, Strong acid hydrolysis, Optimization, Response surface methodology, Cellulose nanocrystals, Cellulose nanofibers, Yield, Properties

\section{Introduction}

Growing demands of the world population are necessitating that we gradually reduce our dependence on nonrenewable petroleum-based materials and transition to

\footnotetext{
${ }^{*}$ Correspondence: jwkim@uark.edu

1 Department of Biological and Agricultural Engineering, University

of Arkansas, Fayetteville, AR 72701, USA

Full list of author information is available at the end of the article
}

green, biomass-based materials that are less detrimental to the environment and human health. Nanomaterials derived from cellulose, the most abundant and renewable biopolymer on earth, have the potential to fill this need (Brinchi et al. 2013; Lee et al. 2014; Postek et al. 2013). Cellulose nanocrystals (CNCs) and cellulose nanofibers (CNFs) have excellent mechanical, optical, rheological and barrier properties. Their biocompatibility and biodegradability makes them useful for diverse applications 
in biomedical, food and cosmetic products, films, coatings, packaging and drug delivery materials, filtration membranes, flexible electronics, and polymer nanocomposites (Brinchi et al. 2013; Dufresne 2013; George and Sabapathi 2015; Habibi et al. 2010; Mishra et al. 2018; Peng et al. 2011; Poletto 2015; Postek et al. 2013; Sinha et al. 2015; Seo et al. 2018). The United States Department of Agriculture (USDA) estimates that the market size of nanocellulose-enabled products will reach $35 \mathrm{M}$ metric tons per year by 2050 (Shatkin et al. 2014). Therefore, cellulosic nanomaterials have huge potential for advancing the bio-based economy. CNCs are rigid, rodlike crystals, with diameters in the range of $5-20 \mathrm{~nm}$ and lengths in the range of $200-500 \mathrm{~nm}$, characterized by high aspect ratio, low density, high tensile strength and stiffness, high surface area and modifiable surface chemistry (Habibi et al. 2010). CNFs have similar diameters but can extend up to a few micrometers in length, resulting in much higher aspect ratios and formation of flexible web-like network structures (Jonoobi et al. 2015). CNCs and CNFs can be extracted from a variety of sources such as tunicates, bacteria and plants, including wood, agricultural residues and industrial crops, using chemical and mechanical processes, respectively. However, wood is the most widely and cheaply available raw material with high cellulose content (Brinchi et al. 2013; Jonoobi et al. 2015; Lee et al. 2014; Sacui et al. 2014).

Utilization of woody biomass for cellulosic nanomaterial synthesis has the potential to add value to the traditional forest products industry (Brinchi et al. 2013), since a substantial infrastructure for planting, harvesting, transporting, debarking, chipping, and pulping different types of wood is already in place (Postek et al. 2013). It can also facilitate the utilization of millions of tons of forestry waste generated in the form of logging residues that are either landfilled or burned in the open, both of which are hazardous to the environment (Hamer 2003). Loblolly pine (Pinus taeda) is one of the most commercially important wood species in the southeastern United States. Its abundance (22.3\% standing tree volume in Arkansas, US), fast growth rate (short rotation of 25-35 years) (Bragg 2011) and existing supply chain for pulp and timber make it an attractive feedstock for commercial scale production of nanocellulose. On the other hand, kraft process is the predominant pulping process for producing cellulose-rich pulp from a variety of hardwood and softwood species (Biermann 1996; Houtman 2018). Kraft pulp could be a sustainable raw material to manufacture nanocellulose, adding to the revenue streams of conventional pulp and paper mills. In a recent report, our group has shown that $\mathrm{CNC}$ yield and crystallinity can be improved by utilizing kraft pulp prepared from pre-extracted loblolly pine (Rajan et al. 2020).
Therefore, in this study, we chose to use pre-extracted loblolly pine kraft pulp as our model substrate for further optimization of CNC yield.

CNCs can be produced from cellulose by a variety of chemical, mechanical and enzymatic methods, often used in combination. Chemical methods make use of strong acids, such as sulfuric, phosphoric, nitric, hydrobromic and hydrochloric acid, or ionic liquids, organic solvents, organic acids and subcritical water to hydrolyze the amorphous regions of cellulose and produce $\mathrm{CNCs}$ (Brinchi et al. 2013; Chen et al. 2016; Novo et al. 2015; Zhang and Liu 2018). On the other hand, mechanical methods, such as bead milling and high-intensity ultrasonication, make use of shear forces to extract CNCs (Amin et al. 2015; Brinchi et al. 2013; Li et al. 2016). However, the concentrated sulfuric acid hydrolysis approach of treating cellulose, initially developed in the 1950s (Battista 1950; Mukherjee and Woods 1953), is still the most common and effective method of producing CNCs from cellulose-rich materials. This method is known to produce stable colloidal dispersions, where particles do not aggregate due to surface charge imparted by sulfate ester groups (Araki et al. 1998; Dong et al. 1998; Lin and Dufresne 2014), and result in higher yields. Several studies aimed at optimizing the sulfuric acid process to maximize $\mathrm{CNC}$ yields from a variety of different raw materials have been published in the past decade. While most commonly tested factors are acid concentration, temperature and hydrolysis duration, some studies have investigated acid-to-pulp ratio, substrate concentration and sonication time too. An important factor that has not been evaluated but could impact $\mathrm{CNC}$ yields, is the raw material particle size. Size reduction techniques, such as milling and grinding, increase the accessible surface area and pore size of biomass; correlation of reduced particle size with the ease of enzymatic digestibility is well documented in biofuels research (Behera et al. 2014). Our study has included the parameter of particle size in its experimental design to assess if $\mathrm{CNC}$ yield improves as a result of reduction in pulp particle size and to determine the optimal particle size for maximizing CNC production.

Depending on the range of conditions tested, the types of raw materials used, and the method of optimization implemented, the effects of independent variables differ, and so do the optimized conditions and corresponding yields. In woody biomass, testing of narrower acid concentration ranges improved CNC yields up to $60-70 \%(\mathrm{w} / \mathrm{w})$ (Dong et al. 2016; Wang et al. 2012, 2014). These studies used bleached hardwood (eucalyptus) kraft pulp and dissolving grade softwood sulfite pulp as raw materials. Optimal conditions and maximal yields reported in these studies may 
not be directly applicable to softwood (e.g., pine) kraft pulp, due to significant differences between hardwood and softwood pulps in terms of chemical composition, anatomical structure and cellulose fiber morphology (Area and Popa 2014; Biermann 1996; Pettersen 1984). Similarly, sulfite and kraft pulps differ from each other in terms of cellulose crystalline structure, fibril aggregation and thermal stability (Hult et al. 2003; Poletto et al. 2011). Therefore, it is imperative that optimized conditions resulting in high CNC yields have to be determined separately for softwood kraft pulp. Highest reported CNC yield from bleached softwood (mixture of cedar, spruce, fir and pine) kraft pulp was only about $30-40 \%$ (Hamad and $\mathrm{Hu} 2010$ ), possibly due to the lack of statistical design of experiments (DOE), leaving much scope for improvement.

In this study, a four-factor, three-level central composite design (CCD) of response surface methodology (RSM) was used as the optimization tool to analyze the effect of four process parameters, i.e., acid concentration, temperature, reaction duration and pulp particle size, on CNC yields. We used fully bleached and highly pure ( $>90 \%$ cellulose) loblolly pine kraft pulp, prepared using an in-house hemicellulose pre-extraction, kraft pulping and bleaching process, as the raw material for CNC production (Rajan et al. 2020). The optimal conditions for sulfuric acid hydrolysis and resulting maximum yields were determined and experimentally validated. Characteristic properties of CNCs, including particle size, crystallinity index and surface charge, were also included as response variables to elucidate how these properties were impacted by a combined set of process conditions, not just by one parameter at a time (Chen et al. 2015; Dong et al. 1998; Hamad and $\mathrm{Hu}$ 2010; Kargarzadeh et al. 2012). This will be helpful to understand which parameters need to be finetuned in the manufacturing process in order to control the quality of CNCs and achieve a desired set of properties. Finally, differences between the properties of $\mathrm{CNCs}$ and the residual, partially hydrolyzed pulp, termed cellulosic solid residues (CSR), were evaluated. Recent optimization studies have suggested that recovering CSR for CNF production has the potential to improve the economic viability of the process (Wang et al. 2012, 2014). Through this work, we aim to determine if recovery of low quantities of CSR at conditions that favor CNC production is practical. Recommendations on the set of conditions that maximize production of either CNCs or CSR (for conversion to CNFs) will be provided. This study paves the way to utilize the commonly available loblolly pine kraft pulp for CNC and CNF production through an optimized strong acid hydrolysis process.

\section{Materials and methods}

\section{Chemicals}

Sulfuric acid ACS grade (95-98\%) was obtained from VWR International (Radnor, PA). Sodium sulfide nonahydrate (98\%) and sodium hydroxide, both ACS grade, were obtained from Beantown Chemical (Hudson, $\mathrm{NH}$ ) and EMD Millipore, Merck (Gibbstown, NJ), respectively. Hydrochloric acid ACS grade (36\%) and granular sodium chlorate (99\%) were obtained from Alfa Aesar (Ward Hill, MA). Hydrogen peroxide (30\%) was obtained from EMD MiiliporeSigma (Burlington, MA).

\section{Pretreatment and bleaching of pinewood}

Loblolly pine (Pinus taeda L.), grown in the School of Forestry and Natural Resources at the University of Arkansas, Monticello, was used in this study. The stem wood was debarked, ground using a Thomas Scientific Wiley Mini-Mill (model 3383-L10, Swedesboro, NJ) and passed through a 20-mesh screen to obtain particles ranging $0.8-0.9 \mathrm{~mm}$ in size. The samples were stored in airtight containers at room temperature until further use. Dilute acid pretreatment of pinewood was conducted for $1 \mathrm{~h}$ at $150^{\circ} \mathrm{C}$ in a $1-\mathrm{L}$ stainless steel bench-top Parr reactor (model 4525, Moline, IL), at solids loading of $20 \% \mathrm{w} / \mathrm{v}$ and sulfuric acid concentration of $0.5 \% \mathrm{w} / \mathrm{w}$ (with respect to dry biomass). After this step, the pretreated biomass was separated from the liquid fraction using vacuum filtration. Kraft pulping of the pretreated biomass was carried out at $170{ }^{\circ} \mathrm{C}$ in the same reactor, at solids loading of $20 \% \mathrm{w} / \mathrm{v}$, effective alkalinity of $24 \%$ and effective sulfidity of $66 \%$. An $\mathrm{H}$-factor of 1500 , which was estimated by the following equation, was used for this reaction.

$$
H=\int_{0}^{t} \exp \left(43.2-\frac{16115}{T}\right) \mathrm{d} t,
$$

where $H$ is $\mathrm{H}$-factor, $t$ is time ( $\min$ ) and $T$ is temperature $\left({ }^{\circ} \mathrm{K}\right)$. After this step, the kraft pulp was separated from the liquid fraction using vacuum filtration. This was followed by two washings with $500 \mathrm{~mL}$ water to remove any residual lignin physically attached to the pulp. Elemental chlorine free (ECF) bleaching of the pulp was carried out in $1000 \mathrm{~mL}$ conical flasks in a water bath at $45^{\circ} \mathrm{C}$ and $70{ }^{\circ} \mathrm{C}$. It consisted of alternating treatments with chlorine dioxide, hydrogen peroxide and sodium hydroxide, until the residual lignin content of the pulp was reduced to $<1 \%(\mathrm{w} / \mathrm{w})$.

\section{Chemical composition analysis}

Chemical composition of raw biomass, i.e., ethanolsoluble extractives, structural carbohydrates and lignin 
content, was measured using National Renewable Energy Laboratory protocols NREL/TP-510-42618 and NREL/ TP-510-42619. The same protocols were used for the estimation of structural carbohydrates in fully bleached kraft pulp as well. However, residual lignin content in bleached kraft pulp was determined based on the Kappa number (K), as given in the T-236-om-99 protocol, published by the Technical Association of the Pulp and Paper Industry (TAPPI) in 1999. Quantification of sugars was done using high performance liquid chromatography (HPLC). The chemical composition of pine biomass and purified pulp, thus determined, is provided in Table 1 . The sequential procedure of dilute acid pretreatment, kraft pulping and ECF bleaching was effective in hemicellulose removal, followed by bulk delignification and removal of residual lignin to less than $1 \%(\mathrm{w} / \mathrm{w})$; highly pure pulp, containing $>90 \%(\mathrm{w} / \mathrm{w})$ cellulose and $<5 \%(\mathrm{w} / \mathrm{w})$ hemicellulose, was obtained at the end of this 3-step treatment process.

\section{CNC synthesis via concentrated acid hydrolysis}

The production of CNCs was performed according to the procedure described by Bondeson et al. (2006) and Wang et al. (2012), with minor alterations. Bleached pulp was first air dried until moisture content was $<5 \%(\mathrm{w} / \mathrm{w})$ and then ground to the desired mesh size using the mini mill. The ground pulp was hydrolyzed at an acid-to-pulp ratio of 8:1 v/w. The reaction was carried out in a 1000$\mathrm{mL}$ beaker placed in a water bath and the solution was constantly mixed at 50 revolutions per minute (RPM) using an overhead stirrer. To stop the reaction, $10 \times$ water was added and the mixture was stirred at 100 RPM for $10 \mathrm{~min}$. The suspension was then centrifuged at $8346 \times g$ for $20 \mathrm{~min}$ and the volume of the supernatant recovered (termed waste stream 1) was recorded. For each sample, three aliquots were stored for HPLC analysis to determine the amount of cellulose and hemicellulose lost in the form

$\begin{aligned} & \text { Table } 1 \text { Chemical composition of pinewood and bleached } \\
& \text { pulp }\end{aligned}$
\begin{tabular}{lcc}
\hline Component & $\begin{array}{l}\text { Pine biomass (\% w/w } \\
\text { o.d.) }\end{array}$ & $\begin{array}{l}\text { Purified } \\
\text { cellulose }^{\mathbf{a}} \text { (\% } \\
\text { w/w o.d.) }\end{array}$ \\
\hline Glucan & $34.8 \pm 0.4$ & $90.8 \pm 0.6$ \\
Xylan & $6.8 \pm 0.1$ & $4.5 \pm 0.0$ \\
Galactan & $2.6 \pm 0.1$ & $1.6 \pm 0.1$ \\
Mannan & $10.0 \pm 1.2$ & $2.1 \pm 0.0$ \\
Lignin & $34.5 \pm 2.0$ & $0.6 \pm 0.1$ \\
Extractives & $14.0 \pm 1.1$ & - \\
Total & $102.8 \pm 0.9$ & $99.0 \pm 0.4$ \\
\hline
\end{tabular}

o.d. oven dry basis

a Purified cellulose was produced in three stages: (1) dilute acid pretreatment, (2) kraft pulping and (3) sequential elemental chlorine free bleaching of monosaccharides, i.e., glucose, xylose, galactose, arabinose, mannose, using NREL/TP-510-42621 protocol. The pellets were recovered and washed with $30 \mathrm{~mL}$ water, vortexed and then centrifuged before discarding the supernatant (termed waste stream 2). The resultant pellet was re-suspended in water and the suspension was dialyzed for two days to remove residual acid and until the $\mathrm{pH}$ reached 7 . The suspension was once again centrifuged to obtain CNCs and CSR as separate streams; CNCs formed a stable suspension in the supernatant, whereas CSR was obtained as pellet. Volumes and weights of each fraction were recorded, and samples were stored at $4{ }^{\circ} \mathrm{C}$ until further use. CNC and CSR yields were determined using the gravimetric method of oven-drying, where samples were dried at $105{ }^{\circ} \mathrm{C}$ until constant weight (Eq. 2). CNC, CSR and total yields as well as carbohydrate losses (Eq. 3) and the overall mass balance, were all expressed as a percentage of the initial dry weight of cellulose pulp.

$$
\begin{aligned}
& \text { Yield }(\%)=\frac{m}{M} \times 100, \\
& \text { Carbohydrate loss }(\%)=\frac{c V}{M} \times 100,
\end{aligned}
$$

where $m$ is the dry weight of total CNC or CSR obtained (g), $M$ is the dry weight of starting material (3 $\mathrm{g}$ of preextracted and bleached loblolly pine kraft pulp), $c$ is the concentration of sugars in waste stream 1 determined using HPLC (recorded in $\mathrm{g} / \mathrm{L}$ ) and $V$ is the total volume of waste stream $1(\mathrm{~L})$.

\section{Optimization of acid hydrolysis parameters}

The Box-Wilson Central Composite Design (CCD) of Response Surface Methodology was used to design a set of experiments, in order to investigate the effect of different acid hydrolysis parameters on $\mathrm{CNC}$ production. Four factors, i.e., acid concentration $(\% \mathrm{w} / \mathrm{w})$, hydrolysis duration (min), hydrolysis temperature $\left({ }^{\circ} \mathrm{C}\right)$ and particle size of pulp (mesh), and three responses, i.e., CNC, CSR and total yields were evaluated. The factors and their corresponding intervals were selected according to in-house exploratory work based on previous reports. The average pulp particle sizes for 20, 40 and 60 mesh sieves corresponded to $0.841 \mathrm{~mm}, 0.420 \mathrm{~mm}$, and $0.250 \mathrm{~mm}$, respectively. The particle size was represented in mesh size instead of $\mathrm{mm}$ for the sake of simplicity in considering equal factor increments for process optimization and will be referred to as such in latter discussions. Each factor had three levels, i.e., low, mid and high, designated as $-1,0$ and 1 , respectively, as illustrated in Table 2 . The ranges were kept neither too narrow nor too wide, so as to prevent exclusion of any optimal conditions while aiming for a strong predictive power of the model. The run order of the trials was randomized 


\begin{tabular}{|c|c|c|c|c|}
\hline \multirow[t]{2}{*}{ Variables } & \multirow{2}{*}{$\begin{array}{l}\text { Orthogonal } \\
\text { scaled factors } \\
\left(X_{\mathrm{i}}\right)\end{array}$} & \multicolumn{3}{|l|}{ Levels } \\
\hline & & -1 (Low) & 0 (Mid) & 1 (High) \\
\hline $\begin{array}{l}\text { Acid concentration } \\
(\% \mathrm{w} / \mathrm{w})\end{array}$ & $x_{1}$ & 54 & 60 & 66 \\
\hline $\begin{array}{l}\text { Hydrolysis duration } \\
\text { (min) }\end{array}$ & $x_{2}$ & 30 & 60 & 90 \\
\hline Temperature $\left({ }^{\circ} \mathrm{C}\right)$ & $x_{3}$ & 45 & 55 & 65 \\
\hline Particle size (mesh) & $X_{4}$ & 20 & 40 & 60 \\
\hline
\end{tabular}

in order to prevent systematic errors and the runs were conducted exactly in the order specified by the software (JMP Pro version 13). The total number of experiments was 26, calculated using the formula: $N=2^{k}$ (factorial points) $+2 k$ (star/axial points) $+r$ (center points), where the number of factors $(k)$ was 4 and the replicates of center point conditions $(r)$ were 2 (Bezerra et al. 2008). Center point conditions $\left(60 \% \mathrm{H}_{2} \mathrm{SO}_{4}, 55^{\circ} \mathrm{C}, 60 \mathrm{~min}\right.$ and $\left.40 \mathrm{mesh}\right)$ were replicated twice, to allow for proper testing of the model's lack of fit (Dean et al. 2017).

\section{Mechanical fibrillation with microfluidizer}

CSR suspensions were diluted to a concentration of $1 \%$ $(\mathrm{w} / \mathrm{v})$ and passed through a low-volume benchtop microfluidizer (Model LV1-UL, Microfluidics, MA, USA) for high shear mechanical fibrillation. Each sample was given three passes through the micron chamber at 16,000 psi (= $11,032 \mathrm{kPa}$ ) to obtain a homogenized CNF suspension.

\section{Characterization of CNCs and CSR \\ TEM imaging}

Aqueous CNC suspensions of $0.025 \%(\mathrm{w} / \mathrm{v})$ concentration were thoroughly vortexed and sonicated and a droplet of the suspension was placed on a carbon coated 300-mesh copper grid. After letting the droplet dry for about $10-15 \mathrm{~min}$, the sample was negatively stained with $1 \%$ uranyl acetate dye for 30-45 s and left to dry overnight. Images were recorded on a JEOL JEM-1011 transmission electron microscope (TEM) operating at $100 \mathrm{kV}$. The attached software had an in-built feature to record the length and width of CNC particles. Morphological measurements of 10-15 CNCs were made per sample for general estimation of the dimensions of CNCs.

\section{$X$-ray diffraction (XRD)}

X-ray diffractometer was used to determine the crystallinity index $(\mathrm{CrI})$ of raw biomass, kraft pulp, CNCs and CNFs, following the Segal method (Segal et al. 1959):

$$
\operatorname{CrI}(\%)=\frac{I_{200}-I_{A M}}{I_{200}} \times 100
$$

where $I_{200}$ is the intensity of the diffraction peak assigned to the plane (200) at $2 \theta=22.7^{\circ}$ and $I_{\mathrm{AM}}$ is the intensity at $2 \theta=18^{\circ}$ coming from the amorphous part of the sample. To prevent the factor of concentration interfering with measurements, the samples used for XRD analysis were air-dried films prepared from solutions of a fixed concentration at $7.5 \mathrm{mg} / \mathrm{mL}$. Raw biomass and kraft pulp were used as is, in powdered form.

\section{ICP-OES analysis}

All CNC and CSR samples were mixed with a solution of $0.8 \mathrm{M}$ nitric acid until the final sample concentration was close to $1 \mathrm{mg} / \mathrm{mL}$. Then the samples were digested for $30 \mathrm{~min}$, centrifuged at $8346 \times \mathrm{g}$ for $10 \mathrm{~min}$, and the supernatant was passed through a $0.2-\mu \mathrm{m}$ nylon syringe filter. Sulfur content of the supernatant was determined using inductively coupled plasma optical emission spectrometry (ICP-OES) technique. All samples were analyzed in triplicate and in-house calibration curves were used to quantify the sulfur content. The data are presented in terms of $\mathrm{mg}$ of $\mathrm{S}$ per $\mathrm{g}$ of $\mathrm{CNC}$ (on dry basis).

\section{Dynamic light scattering (DLS)}

Characteristics, such as Z-average diameter or particle size (length), dispersity $(\boxplus)$ and zeta-potential, of $\mathrm{CNC}$ and CSR suspensions at $0.025 \%(\mathrm{w} / \mathrm{v})$ concentration, were measured in triplicate using the Malvern Zeta Sizer Nano ZS (model ZEN3600, Worcestershire, UK). Samples were sonicated in an ultrasonic water bath for $5 \mathrm{~min}$ before analysis.

\section{Results and discussion Dependence of CNC-to-CSR ratio on hydrolysis severity} The concept of simultaneously recovering $\mathrm{CNCs}$ and CSR (cellulosic solid residue) to minimize cellulose loss and increase overall yield was first introduced by Wang et al. (2012). Separation is achieved using centrifugation of acid-hydrolyzed and dialyzed pulp, where CNC particles remain suspended in the supernatant and CSR, consisting of fibers bigger in size and less sulfated than CNCs, settles down in the form of a pellet. Of the 26 experimental runs conducted in our study, both CNCs and CSR were recovered in only 13 runs, whereas for the rest, the CSR fraction was below recoverable limits. In general, depending on the severity of reaction conditions, CNC yields varied between $0 \%$ and $52 \%$, and CSR yields varied between $0 \%$ and as high as $85 \%$ (Table 3 ). The technique of cluster analysis was used to condense the yield data into smaller, more coherent groups, where each group represented similar yields. The data were 
first standardized and k-means clustering approach was used ( $\mathrm{R}$ code and detailed results provided in supplementary data) to identify the optimal number of clusters for appropriate data classification (Fig. 1).

Cluster 1 consisted of three runs (21, 22 and 24), characterized by very high CSR yields (78-85\%), extremely low CNC yields $(<3 \%)$, and negligible carbohydrate losses $(<5 \%)$ (Table 3). CSR recovered in these runs was barely hydrolyzed and was more or less similar in texture to the original pulp, making it unsuitable for microfluidization. It could be attributed to the low severity of reaction conditions, i.e., a combination of low acid concentration $(54 \%)$ and low temperature $\left(45^{\circ} \mathrm{C}\right)$. Acid concentrations below $58 \%$ have been reported to cause insufficient cellulose depolymerization and result in low $\mathrm{CNC}$ yields (Wang et al. 2014). CNCs and CSR from these three runs were not included in further characterization.

Cluster 2 consisted of five runs (7, 9, 11, 12 and 17) characterized by relatively more CSR than CNC yields and minimal losses ranging between 4 and $8 \%$ (Table 3 ). Three of these runs $(9,12$ and 17) had particularly high CSR and total (CNC + CSR) yields that averaged at $60 \%$ and $75 \%$, respectively. These conditions were also favorable for hydrolyzing the CSR just enough to facilitate subsequent mechanical fibrillation, without substantial conversion into $\mathrm{CNCs}$ and/or degradation into sugars. If the goal is to produce CNFs, the conditions of run 17 , i.e., $54 \%$ acid, $90 \mathrm{~min}, 45^{\circ} \mathrm{C}$ and 20 mesh, will be more suitable because of high yields obtained with a conservative use of acid and energy (lower temperature and reduced milling requirements). With increasing reaction severity, the gap between CSR and CNC yields was reduced due to increasing hydrolysis of CSR into CNCs. This was concomitant with a simultaneous increase in cellulose degradation (12-15\%) and a total lower nanocellulose yield (50-65\%), evident from the other two runs (7 and 11) in this cluster.

Table 3 RSM-CCD experimental design matrix and response data

\begin{tabular}{|c|c|c|c|c|c|c|c|c|c|}
\hline \multirow{2}{*}{$\begin{array}{l}\text { Run no. } \\
\text { (cluster no.) }\end{array}$} & \multicolumn{4}{|c|}{ Factors } & \multicolumn{3}{|l|}{ Responses } & \multicolumn{2}{|c|}{ Mass balance } \\
\hline & $X_{1}, \%$ & $x_{2}, \min$ & $X_{3},{ }^{\circ} \mathrm{C}$ & $X_{4}$, Mesh size & CNC yield, \% & CSR yield, \% & Total yield, \% & Loss, $\%$ & $\begin{array}{l}\text { Overall } \\
\text { closure, \% }\end{array}$ \\
\hline $1(3)$ & 54 & 90 & 65 & 20 & $27.9 \pm 1.3$ & - & $27.9 \pm 1.3$ & 25.6 & 53.5 \\
\hline $2(3)$ & 66 & 30 & 65 & 20 & $25.2 \pm 0.9$ & - & $25.2 \pm 0.9$ & 29.2 & 54.4 \\
\hline $3(2)$ & 54 & 30 & 65 & 20 & $33.7 \pm 2.8$ & $22.0 \pm 2.2$ & $55.7 \pm 0.6$ & 18.6 & 74.3 \\
\hline $4(2)$ & 60 & 60 & 55 & 40 & $38.3 \pm 2.9$ & $16.9 \pm 3.5$ & $55.2 \pm 0.6$ & 18.5 & 73.7 \\
\hline $5(2)$ & 54 & 30 & 65 & 60 & $41.7 \pm 1.9$ & $20.7 \pm 0.0$ & $62.4 \pm 1.9$ & 10.4 & 72.8 \\
\hline $6(2)$ & 60 & 60 & 55 & 60 & $52.1 \pm 3.3$ & - & $52.1 \pm 3.3$ & 19.9 & 72.0 \\
\hline $7(1)$ & 66 & 30 & 45 & 20 & $20.1 \pm 0.5$ & $31.4 \pm 0.6$ & $51.5 \pm 0.0$ & 11.5 & 63.0 \\
\hline $8(2)$ & 60 & 90 & 55 & 40 & $51.6 \pm 1.6$ & - & $51.6 \pm 1.6$ & 15.9 & 67.5 \\
\hline $9(1)$ & 60 & 60 & 45 & 40 & $12.8 \pm 0.4$ & $62.3 \pm 1.5$ & $75.1 \pm 1.2$ & 6.3 & 81.4 \\
\hline $10(2)$ & 54 & 90 & 65 & 60 & $37.2 \pm 1.1$ & - & $37.2 \pm 1.1$ & 15.0 & 52.2 \\
\hline $11(1)$ & 60 & 30 & 55 & 40 & $29.5 \pm 0.9$ & $33.6 \pm 3.9$ & $63.0 \pm 3.1$ & 14.9 & 77.9 \\
\hline $12(1)$ & 54 & 60 & 55 & 40 & $23.2 \pm 0.2$ & $53.0 \pm 2.6$ & $76.2 \pm 2.4$ & 7.7 & 83.9 \\
\hline $13(3)$ & 66 & 30 & 65 & 60 & $15.0 \pm 0.6$ & - & $15.0 \pm 0.6$ & 34.8 & 49.8 \\
\hline $14(2)$ & 60 & 60 & 55 & 40 & $37.1 \pm 1.6$ & $20.8 \pm 1.8$ & $57.9 \pm 0.2$ & 15.0 & 72.9 \\
\hline $15(3)$ & 66 & 90 & 65 & 20 & $22.6 \pm 0.3$ & - & $22.6 \pm 0.3$ & 37.5 & 60.1 \\
\hline $16(3)$ & 66 & 60 & 55 & 40 & $17.3 \pm 0.4$ & - & $17.3 \pm 0.4$ & 43.6 & 60.9 \\
\hline $17(1)$ & 54 & 90 & 45 & 20 & $15.6 \pm 0.4$ & $59.6 \pm 1.3$ & $75.2 \pm 0.9$ & 4.4 & 79.6 \\
\hline $18(3)$ & 66 & 90 & 65 & 60 & $23.4 \pm 0.8$ & - & $23.4 \pm 0.8$ & 33.3 & 56.7 \\
\hline $19(2)$ & 60 & 60 & 55 & 20 & $38.0 \pm 0.9$ & $13.6 \pm 0.3$ & $51.5 \pm 0.6$ & 21.8 & 73.3 \\
\hline $20(3)$ & 66 & 30 & 45 & 60 & $18.0 \pm 0.2$ & - & $18.0 \pm 0.2$ & 23.5 & 41.5 \\
\hline $21(4)$ & 54 & 30 & 45 & 20 & $0.6 \pm 0.1$ & $83.3 \pm 2.3$ & $83.9 \pm 2.3$ & 1.6 & 85.5 \\
\hline $22(4)$ & 54 & 90 & 45 & 60 & $3.2 \pm 0.0$ & $77.7 \pm 0.8$ & $80.8 \pm 0.8$ & 3.5 & 84.3 \\
\hline $23(2)$ & 60 & 60 & 65 & 40 & $33.2 \pm 1.3$ & - & $33.2 \pm 1.3$ & 24.3 & 57.5 \\
\hline $24(4)$ & 54 & 30 & 45 & 60 & $1.1 \pm 0.0$ & $84.5 \pm 1.0$ & $85.6 \pm 0.9$ & 2.2 & 87.8 \\
\hline $25(3)$ & 66 & 90 & 45 & 20 & $11.9 \pm 0.1$ & - & $11.9 \pm 0.1$ & 40.5 & 52.4 \\
\hline $26(3)$ & 66 & 90 & 45 & 60 & $14.8 \pm 0.3$ & - & $14.8 \pm 0.3$ & 44.9 & 59.7 \\
\hline
\end{tabular}

\% Total yield is the sum of CNC and CSR yields. \% Loss is the total carbohydrate (cellulose and hemicellulose) lost in the first waste stream in the form of monosaccharides. Yields, losses and overall mass closure are expressed as a $\%$ of the initial dry weight of kraft pulp 


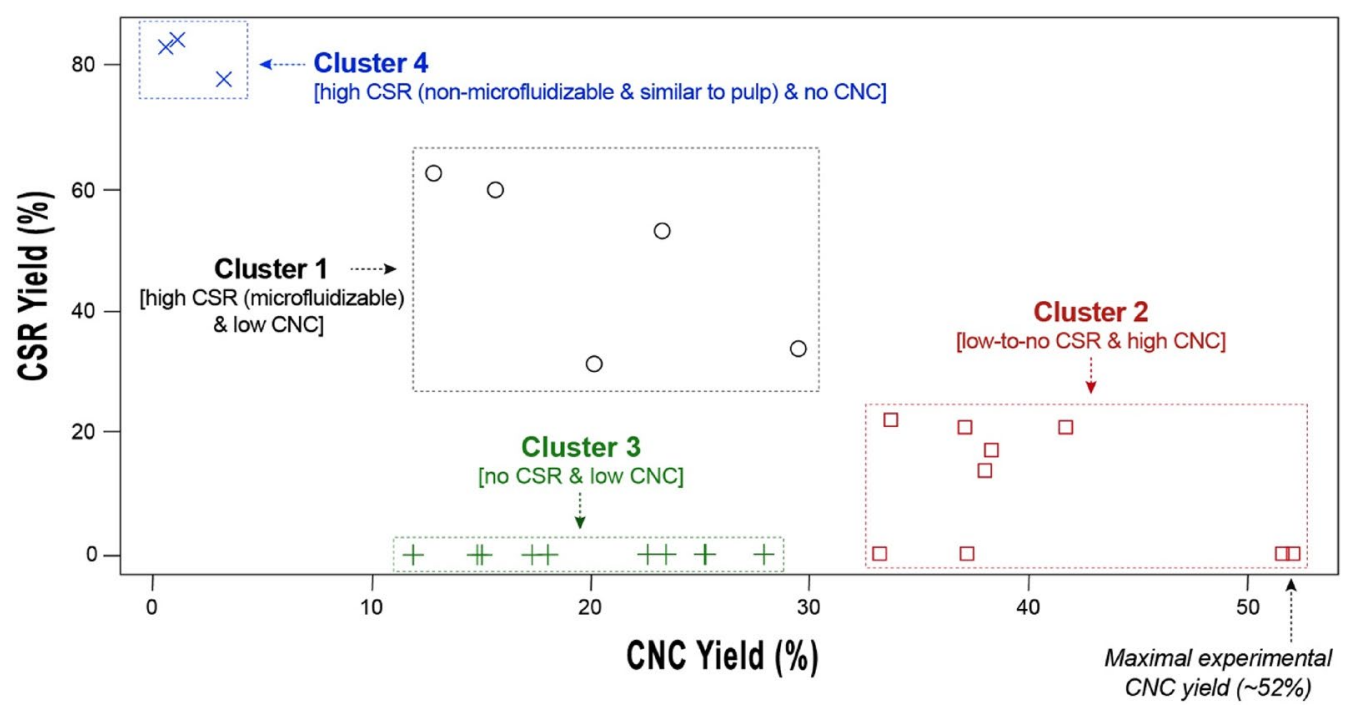

Fig. 1 Cluster analysis of experimental CNC and CSR yields

Cluster 3 consisted of nine runs (3, 4, 5, 6, 8, 10, 14, 19 and 23) characterized by relatively more CNCs than CSR yields; cellulose lost in the waste stream ranged between $10-25 \%$ and total nanocellulose yield ranged between 52 and $62 \%$. Five of these runs $(3,4,5,14$ and 19), including center point conditions ( 4 and 14), yielded $34-42 \%$ CNCs and 14-22\% CSR indicative of the reaction leaning toward CNC formation. The exceptions were runs 10 and 23 , where high temperatures caused complete conversion of pulp into CNCs, with yields ranging between $30-40 \%$ and negligible CSR recovery, pointing towards the dominant effect of temperature in cellulose depolymerization kinetics (Dong et al. 2016; Wang et al. 2014). Highest CNC yields (52\% CNC and no CSR) were obtained in two runs (6 and 8), which differed from center point conditions by longer hydrolysis duration or smaller particle size (higher mesh).

Cluster 4 consisted of nine runs $(1,2,13,15,16,18,20$, 25 and 26) characterized by very low CNC yields ranging between $10-30 \%$ and no CSR recovery, indicating deleterious cellulose hydrolysis. It was observed that most of these runs were conducted at the high acid concentration of $66 \%$, which caused high cellulose degradation and resulted in low yields (Table 3), thus indicating the dominant effect of acid concentration on cellulose depolymerization kinetics as reported in previous studies (Hamad and Hu 2010; Wang et al. 2012, 2014). The only exception to this was run 1 that yielded $28 \%$ CNCs even at the low acid concentration of $54 \%$, possibly due to the combination of longer reaction duration with high temperature. But the general trend agrees with previous studies, where $62-65 \% \mathrm{H}_{2} \mathrm{SO}_{4}$ resulted in $\mathrm{CNC}$ yields of 30-45\% from microcrystalline cellulose (MCC), softwood kraft pulp and Kenaf pulp (Bondeson et al. 2006; Hamad and Hu 2010; Kargarzadeh et al. 2012; Ngwabebhoh et al. 2018). Acid concentrations above $60 \%$ should be avoided in CNC production as these lead to degradation into monomeric and oligomeric sugars that cannot be economically recovered and utilized (Wang et al. 2012, 2014). The ideal acid concentration range is $58-62 \%$ as per Wang et al. (2014). Overall, the experimental data indicated that, potential for maximizing CNC yields from pine kraft pulp lies in optimizing around the parameters of $54-60 \%$ acid, $55-65{ }^{\circ} \mathrm{C}, 60-90 \mathrm{~min}$ and $40-60$ mesh; however, some degradation of pulp (10-20\%) is bound to take place as a result of these processing conditions.

\section{Regression analysis}

The following second-order polynomial equation was used to (a) determine significant model terms and (b) develop an empirical model correlating the response, i.e., $\mathrm{CNC}$ yield to the four independent variables under investigation. Results of analysis of variance (ANOVA) are given in Table 4.

$$
Y=\beta_{0}+\sum_{i} \beta_{i} x_{i}+\sum_{i i} \beta_{i i} x_{i}^{2}+\sum_{i j} \beta_{i j} x_{i} x_{j}+\varepsilon,
$$

where $\beta_{0}$ is the constant co-efficient, $\beta_{i}$ is the linear effect of the main factor $x_{i}, \beta_{i j}$ is the linear-by-linear interaction effect of the input factors $x_{i}$ and $x_{j}, \beta_{i i}$ is the quadratic effect of the input factor $x_{i}$ and $Y$ is the response. Based on non-linear regression analysis of the response data, the following quadratic model (Eq. 6) was obtained. The 
Table 4 ANOVA for response surface quadratic model

\begin{tabular}{|c|c|c|c|c|c|}
\hline Source & Sum of squares & $d f$ & Mean square & F value & $\begin{array}{l}p \\
\text { Prob }>\text { F }\end{array}$ \\
\hline Model & 4309.22 & 14 & 307.80 & 5.44 & 0.0038 \\
\hline Intercept & & & & & $<0.0001$ \\
\hline$x_{1}$ & 14.05 & 1 & 14.05 & 0.25 & 0.6280 \\
\hline$x_{2}$ & 30.16 & 1 & 30.16 & 0.53 & 0.4805 \\
\hline$x_{3}$ & 1454.40 & 1 & 1454.40 & 25.72 & 0.0004 \\
\hline$X_{4}$ & 6.60 & 1 & 6.60 & 0.12 & 0.7390 \\
\hline$x_{1} x_{2}$ & 9.61 & 1 & 9.61 & 0.17 & 0.6881 \\
\hline$x_{1} x_{3}$ & 607.62 & 1 & 607.62 & 10.75 & 0.0074 \\
\hline$X_{1} X_{4}$ & 12.25 & 1 & 12.25 & 0.22 & 0.6507 \\
\hline$x_{2} x_{3}$ & 6.50 & 1 & 6.50 & 0.12 & 0.7409 \\
\hline$x_{2} X_{4}$ & 1.21 & 1 & 1.21 & 0.02 & 0.8863 \\
\hline$X_{3} X_{4}$ & 22.56 & 1 & 22.56 & 0.40 & 0.5405 \\
\hline$x_{1}^{2}$ & 681.02 & 1 & 681.02 & 12.04 & 0.0052 \\
\hline$x_{2}^{2}$ & 40.83 & 1 & 40.83 & 0.72 & 0.4136 \\
\hline$x_{3}^{2}$ & 470.70 & 1 & 470.70 & 8.32 & 0.0148 \\
\hline$X_{4}^{2}$ & 184.72 & 1 & 184.72 & 3.27 & 0.0981 \\
\hline Total error & 622.01 & 11 & 56.55 & & \\
\hline Lack of fit & 621.29 & 10 & 62.13 & 86.29 & 0.0836 \\
\hline Pure error & 0.72 & 1 & 0.72 & & \\
\hline Grand total & 4931.23 & 25 & & & \\
\hline
\end{tabular}

Italic values indicate significance of $p$ value $(p<0.05)$

positive coefficient values signify synergistic effects that increase $\mathrm{CNC}$ yield and the negative coefficient values signify antagonistic effects that decrease $\mathrm{CNC}$ yield:

$$
\begin{aligned}
\operatorname{CNC} \text { yield }(\%)= & 36.84-0.88 \times\left(\frac{X_{1}-60}{6}\right) \\
& +8.99 \times\left(\frac{X_{3}-55}{10}\right)-6.16 \times\left(\frac{X_{1}-60}{6}\right) \\
& \times\left(\frac{X_{3}-55}{10}\right)-16.31 \times\left(\frac{X_{1}-60}{6}\right)^{2} \\
& -13.56 \times\left(\frac{X_{3}-55}{10}\right)^{2} .
\end{aligned}
$$

At $p<0.05$, the quadratic model was found to be significant and the model process parameters that had a significant effect on CNC yield were the linear effect of temperature $\left(X_{3}\right)$, quadratic effects of temperature $\left(X_{3}^{2}\right)$ and acid concentration $\left(X_{1}^{2}\right)$, and the interaction effect of acid concentration and temperature $\left(X_{1} X_{3}\right)$. Even though the linear effect of acid concentration $\left(X_{1}\right)$ was not significant, the term appears in the equation because it was contained in the interaction effect $\left(X_{1} X_{3}\right)$, which was significant. Remaining model terms, including main, quadratic and interaction effects of hydrolysis duration and particle size, did not have a significant effect on CNC yield. As mentioned before, various studies have reported

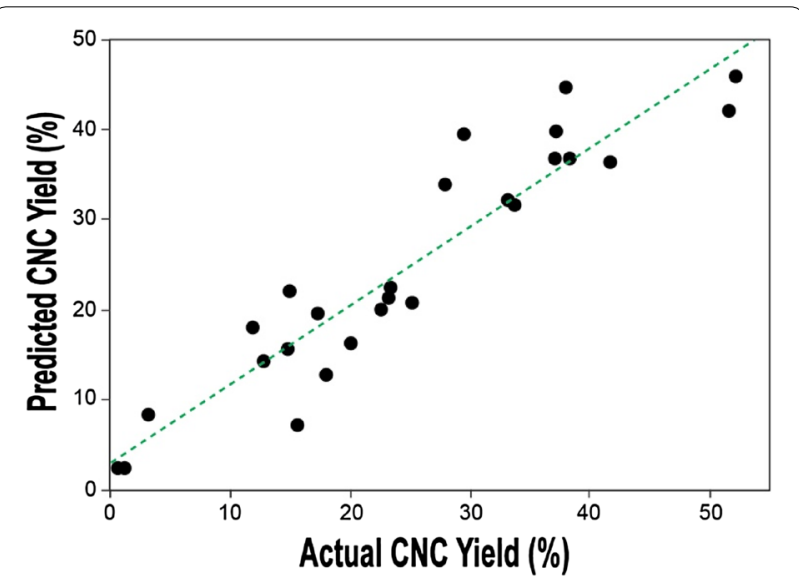

Fig. 2 Experimental vs predicted yields in the regression model

acid concentration, temperature, as well as hydrolysis duration, to have a significant effect on the yield of CNCs. However, the hydrolysis duration was not found significant in our study, which could partly be attributed to (a) the relatively narrower range tested, and (b) the fact that for a given combination of acid concentration and temperature, the bulk of $\mathrm{CNC}$ generation seemed to occur within the first $30 \mathrm{~min}$ of the reaction and did not seem to change significantly with further increase in hydrolysis duration. Contrary to our hypothesis, the increase in surface area and cellulose accessibility with the reduction in pulp particle size from 20 to 60 mesh did not cause significant changes in $\mathrm{CNC}$ yields. A previous study has also reported that depolymerization of cellulose during acid hydrolysis was strongly correlated to the crystallinity index of the pulp rather than its particle size (Lacerda et al. 2013). It is possible that all pulp mesh sizes had similar crystallinity indices and hence produced similar CNC yields.

Insignificance of the "lack of fit" test indicated that the regression model adequately described the relationship between the independent variables tested and the response variable. In models involving multiple independent variables, $R_{\text {adj }}^{2}$ is a more robust estimation of "goodness of fit" than $\mathrm{R}^{2} . R_{\text {adj }}^{2}$ was 0.71 , implying $71 \%$ of the variation in data was explained by the regression model and indicating a good fit between the model and experimental data (Fig. 2). Root mean square error (RMSE), a measure of unexplained variance, indicates the proximity of experimental values to predicted values. Lower values of RMSE indicate better fit; RMSE of the above model was 4.76, indicating the range of deviation of experimental yields from predicted yields is \pm 4.76 . Acceptable values of both $R_{\text {adj }}^{2}$ and RMSE were indicative of good accuracy of the model in predicting $\mathrm{CNC}$ yields within the experimental ranges tested in this study. 


\section{Surface plots, optimization and model verification}

Three-dimensional response surface plots and corresponding contour plots constructed using Eq. 6 are given in Fig. 3. These plots depicted the combined effects of two factors on CNC yield, while other factors were kept constant at their medium levels. Elliptical contour plots imply that the interaction between the variables is significant and circular contour plots mean that the interaction between the variables is not important (Lu et al. 2013). Contour plots of acid concentration vs temperature and duration vs particle size were elliptical, indicating significance of these interactions (Additional file 1: Fig. S1). However, ANOVA results indicated that only the interaction of acid concentration and temperature was significant. A possible reason for this discrepancy is that CNC yield was not as drastically affected with alterations in duration and particle size as with alterations in acid concentration and temperature, because the yields only changed from 38 to $55 \%$ vs 0 to $38 \%$, respectively (Fig. 3a, e). Moreover, when acid concentration and temperature levels were at their extreme, the $\mathrm{CNC}$ yields remained low regardless of the particle size or hydrolysis duration (Fig. 3b-d, f), thus proving the importance of acid concentration $\times$ temperature interaction effect for maximizing $\mathrm{CNC}$ yield. All other interactions were insignificant as per ANOVA (Table 4) as well as the contour plots (Additional file 1: Fig. S1).

The predicted optimal hydrolysis conditions were acid concentration of $59.5 \%$, temperature of $58.5{ }^{\circ} \mathrm{C}$, hydrolysis duration of $56 \mathrm{~min}$ and pulp particle size of 40 mesh. Corresponding predicted CNC and CSR yields were $38.4 \%$ and $16.6 \%$, respectively, totaling $55 \%$ of total nanocellulose yield. These predicted values were verified experimentally. CNC and CSR yields at these exact conditions were found to be $39.7 \%$ and $15 \%$, respectively, totaling $54.7 \%$ of nanocellulose yield (Table 5). This is in very good agreement with the modeled results, i.e., within $95 \%$ confidence interval, thus verifying the adequacy and accuracy of the model. The results were in line with previous findings where overall nanocellulose yield was improved by using acid concentration ranging between 58 and $62 \%$ and a moderate temperature ranging between 50 and $60{ }^{\circ} \mathrm{C}$, and by recovering both $\mathrm{CNC}$ and CSR streams (Wang et al. 2012, 2014).

By using slightly altered optimal conditions of $60 \%$ acid, $58{ }^{\circ} \mathrm{C}, 60 \mathrm{~min}$ and 40 mesh particle size, up to $60 \% \mathrm{CNC}$ yield (with no leftover CSR) was obtained from loblolly pine (softwood) kraft pulp. This approached maximum yields reported in previous studies from eucalyptus (hardwood) kraft pulp (Wang et al. 2012) and softwood sulfite pulp (Dong et al. 2016; Wang et al. 2012), although
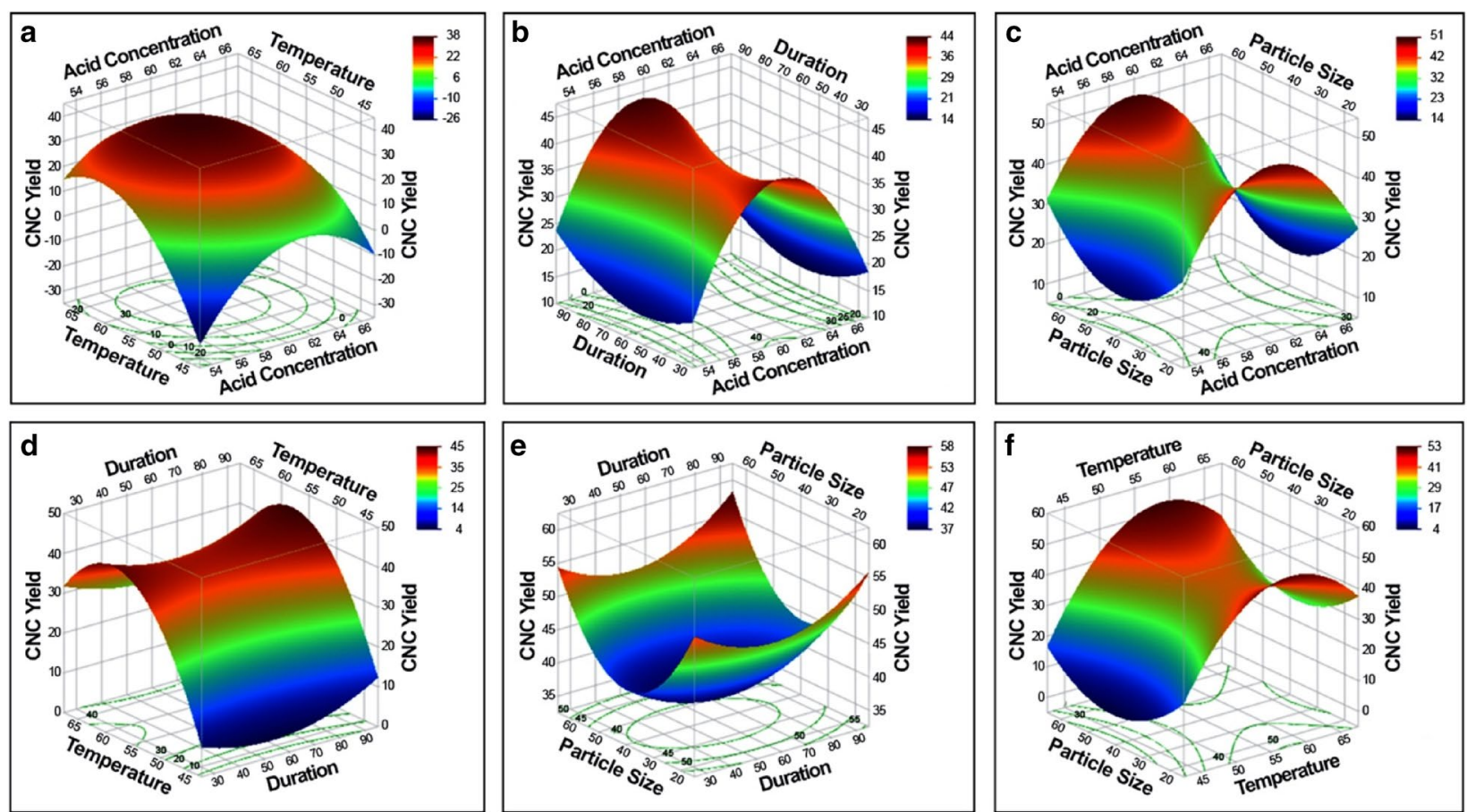

Fig. 3 Response surface and contour plots showing interaction effects of different process parameters on CNC yield: a acid concentration $\times$ temperature; $\mathbf{b}$ acid concentration $\times$ hydrolysis duration; $\mathbf{c}$ acid concentration $\times$ particle size; $\mathbf{d}$ temperature $\times$ hydrolysis duration; $\mathbf{e}$ particle size $\times$ hydrolysis duration, and $\mathbf{f}$ particle size $\times$ temperature 
Table 5 Evaluation of yields and properties of CNCs and CSR at optimum conditions

\begin{tabular}{llllll}
\hline & Predicted yield (\%) & Experimental yield (\%) & Z-average size $(\mathbf{n m})$ & $\boldsymbol{D}$ & Zeta-potential (mV) \\
\hline CNC & 38.4 & $39.7 \pm 0.5$ & $162.8 \pm 5.4$ & $0.43 \pm 0.01$ & $-47.8 \pm 0.9$ \\
CSR & 16.6 & $15.0 \pm 0.2$ & $270.4 \pm 2.8$ & $0.68 \pm 0.15$ & $-43.9 \pm 4.6$ \\
Total & 55.0 & $54.7 \pm 0.3$ & - & - & - \\
\hline
\end{tabular}

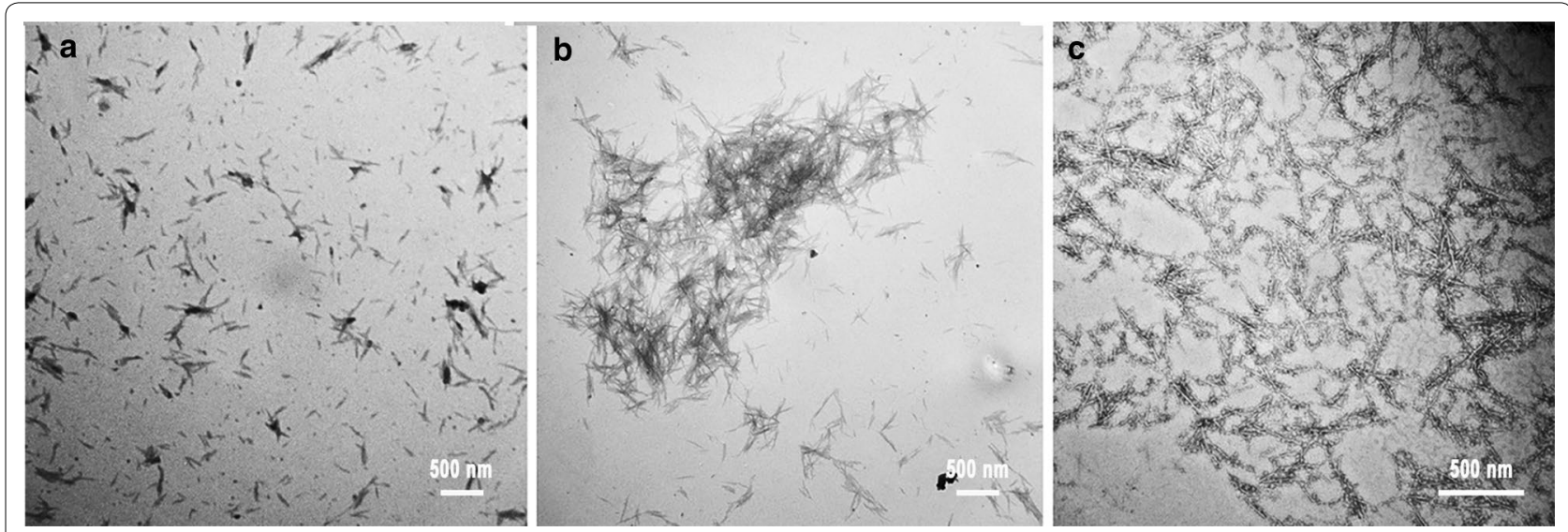

Fig. 4 TEM images of a CNC at optimal conditions, b CSR at optimal conditions, and c CNF (or microfluidized CSR) from high CSR yielding conditions (i.e., run $17-54 \% \mathrm{w} / \mathrm{w}$ acid, $45^{\circ} \mathrm{C}$, 90 min, 20 mesh)

the reaction duration was significantly lower in our study (60 min vs $100-200 \mathrm{~min}$ ), which indicates the need to optimize this factor for each raw material. It should be noted that yields among different studies are often not directly comparable due to variations in process conditions such as impeller RPM and acid-to-pulp ratio, differences in the method of yield measurement, the way yield is defined or expressed, the composition and purity of starting material and the fact that some studies separate CNCs from CSR while others do not. Lastly in literature, the acid-to-pulp ratio used during acid hydrolysis varied from 1:8 g/mL (Wang et al. 2012) and 1:10 g/mL (Bondeson et al. 2006) to $1: 20 \mathrm{~g} / \mathrm{mL}$ (Benini et al. 2018). This implies that acid-to-pulp ratio could be an important parameter that impacts hydrolysis kinetics and should be investigated and standardized in future work.

\section{Justification of CSR recovery at optimal conditions}

We characterized CNCs and CSR obtained at optimal conditions (Table 5), in order to gain an understanding of the differences in their key properties and to determine if it is necessary to separate the two streams at these conditions. DLS analysis showed that the average dispersity of CNCs was roughly 0.4 , whereas that of CSR approached 0.7. Average particle size of CNCs ranged between 160 and $170 \mathrm{~nm}$, and that of CSR was roughly $270 \mathrm{~nm}$. $D$ values, in addition to TEM images (Fig. 4a, b) and particle

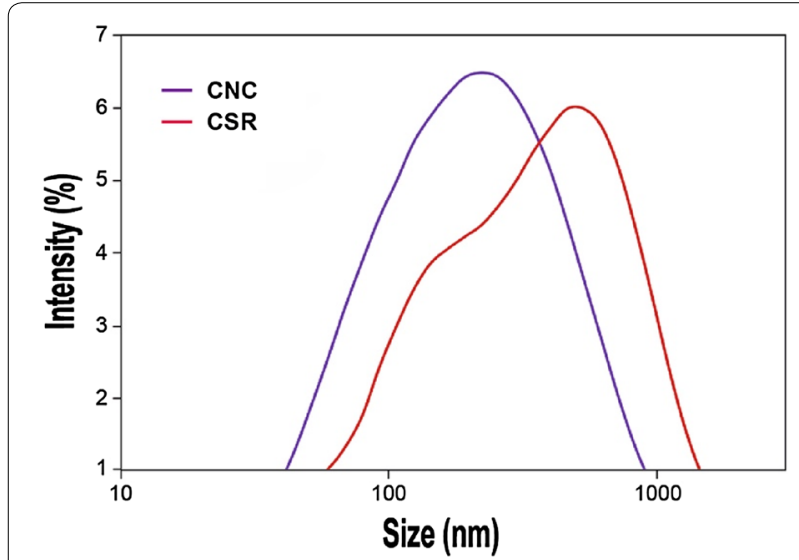

Fig. 5 Particle size distribution of CNC and CSR at optimal conditions (59.5\% w/w acid, $58.5^{\circ} \mathrm{C}, 56 \mathrm{~min}, 40$ mesh). Each spectrum is an average of three replicates

size distribution curves (Fig. 5), indicated that the CNC fraction consisted of nanocrystals with a more uniform size distribution. On the other hand, CSR consisted of a heterogeneous distribution of nanoparticles, both crystals and short fibers, which possibly aggregated and separated from CNCs during centrifugation. The two fractions had quite similar surface charge, as the average zeta-potential of CNCs and CSR was $-48 \mathrm{mV}$ and 
$-44 \mathrm{mV}$, respectively. But whether CSR should be recovered and processed as a separate stream when using optimal conditions is debatable, because it translates into adding two unit-operations (centrifugation and mechanical fibrillation) to the process, for a small amount of CSR that's approaching CNCs in its physicochemical properties. CNCs are valued for their optical, mechanical and rheological properties, and the ability to be chemically functionalized (Moon et al. 2011). Therefore, mixing CNCs with CSR at the pretext of maximizing nanocellulose yield could reduce their commercial value for certain applications. On the other hand, recovering the two fractions separately could add to the revenue streams via production of CNFs. Future techno-economic evaluation of costs involved in the energy-intensive microfluidization operations, for different $\mathrm{CNC} / \mathrm{CNF}$ yield scenarios, is required to validate the benefits of separating the CSR stream.

Our recommendation would be to further optimize the best conditions identified in this study to facilitate complete conversion of cellulose into CNCs and eliminate the need to recover CSR without sacrificing the yield. This can be achieved by using $58-60 \%$ acid concentration to treat pulp of 40 mesh particle size at $55-60{ }^{\circ} \mathrm{C}$ for 60-75 min. By doing so, it may be possible that well-dispersed and colloidally stable $(-40$ to $-50 \mathrm{mV})$ suspensions, containing $\mathrm{CNCs}$ with particle length $<200 \mathrm{~nm}$ and moderate dispersity (0.3-0.5), are obtained with yields ranging between $55-60 \%$. The same process could be altered by using $50-55 \%$ acid concentration to treat pulp of 20 mesh particle size at $45^{\circ} \mathrm{C}$ for $30-45 \mathrm{~min}$ to obtain high yields of partially hydrolyzed but adequately sulfated pulp, that could easily be defibrillated into nanofibers with lower energy requirements compared to untreated pulp (Börjesson and Westman 2015). Recovering and processing higher quantities of CSR at these milder conditions could be economically more beneficial than implementing those steps at harsher severities that favor higher $\mathrm{CNC}$ yields.

\section{Effect of processing conditions on properties of CNCs and CSR \\ Crystallinity index}

Crystallinity of cellulose affects its strength and stiffness; it can have a direct impact on the mechanical properties of nanocomposites that have CNCs and CNFs as fillers (Ahvenainen et al. 2016). Crystallinity index (CrI) is a parameter that quantifies the relative amount of crystalline material present in a cellulosic sample. XRD diffraction patterns of pine biomass, bleached kraft pulp, CNCs from run 4 (high CNC yielding conditions) and CNFs from run 17 (high CSR yielding conditions), are given in Fig. 6. The diffractograms were representative of the semi-crystalline structure of cellulose $\mathrm{I} \beta$, the polymorph found in higher plants (Thygesen et al. 2005), which is characterized by crystalline peaks at $15^{\circ}, 16.5^{\circ}, 22.5^{\circ}$ and $34.5^{\circ}$ corresponding to the crystallographic planes of (1-10), (110), (200) and (004), respectively. The intensity at $18^{\circ}$ represents contribution of the amorphous fraction.

CrI, calculated using Eq. (4) was $45 \%$ for biomass and increased to $74 \%$ for pulp due to the removal of amorphous lignin, hemicellulose and extractive fractions. CrI ranged between 75 and $80 \%$ for CNCs and CNFs from all conditions (Table 6). CrI of CNFs was slightly higher than that of corresponding CNCs, possibly due to higher crystallite size (Nam et al. 2016), indicating lower impact of acid hydrolysis on the crystalline structure of CNFs. No correlation was found between $\mathrm{CrI}$ of $\mathrm{CNCs}$ and any of the four process parameters investigated in this study. This was supported by ANOVA (Additional file 1: Table S1), where none of the factors was found to have a significant effect on CrI. In line with previous reports (Chen et al. 2015; Kargarzadeh et al. 2012), a slight increase in CrI with increasing acid concentration and hydrolysis duration was observed in our study; however, these changes were not significant at $p<0.05$. Overall, our results indicated that the crystallinity of CNCs was preserved regardless of the conditions used to produce them (within the experimental ranges tested in this study). However, small differences in CrI can be attributed to minor changes in crystallite size (Nam et al. 2016), which are bound to take place as reaction severity changes. We would like to note that even though Segal method is the most commonly used method, it has received criticism for its inaccurate and unrealistic estimation of crystallinity because of reasons mentioned in detail elsewhere (Park et al. 2010). Methods using area under the curve and peak fitting are more appropriate for accurate determination of crystallinity; however, Segal method was chosen because it can reliably be used to determine relative differences in $\mathrm{CrI}$ among samples of the same type (Ahvenainen et al. 2016).

\section{Sulfur content and zeta-potential}

Surface charge density, arising from sulfate ester groups imparted during sulfuric acid hydrolysis, is a critical characteristic of CNCs. It can have a crucial influence on the surface chemistry and accessibility of CNCs for chemical modifications, and it impacts their physical properties too, such as colloidal stability, thermal degradation, birefringence and chiral nematic behavior, all of which are significant for different applications (Lin and Dufresne 2014; Reid et al. 2017). Many studies indicate that degree of sulfation of CNCs is strongly dependent on hydrolysis conditions, such as hydrolysis duration, acid concentration, acid-to-pulp ratio and temperature 


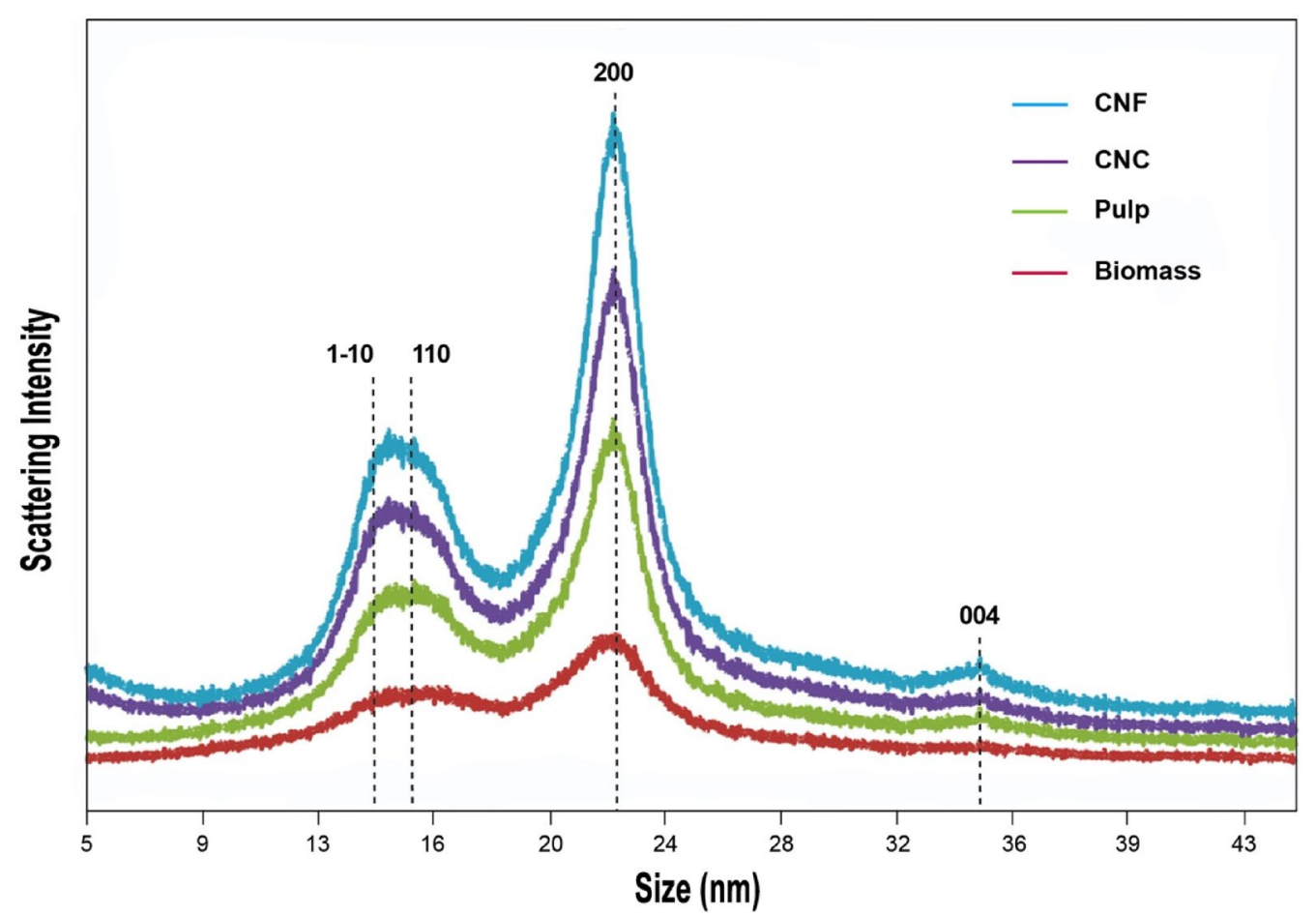

Fig. 6 XRD diffraction patterns of original biomass (red line), bleached Kraft pulp (green line), CNCs (purple line) from high CNC yielding conditions (i.e., run 4-60\% w/w acid, $60 \mathrm{~min}, 55^{\circ} \mathrm{C}, 40$ mesh) and CNFs (cyan line) from high CSR yielding conditions (i.e., run $17-54 \% \mathrm{w} / \mathrm{w}$ acid, $45^{\circ} \mathrm{C}, 90 \mathrm{~min}$, 20 mesh)

(Bondeson et al. 2006; Chen et al. 2015; Dong et al. 1998; Hamad and Hu 2010; Kargarzadeh et al. 2012; Lin and Dufresne 2014; Selim et al. 2005; Wang et al. 2012); however, it was also suggested that $\mathrm{S}$ content may not be a good measure of hydrolysis severity. It should be noted that, in the literature, the degree of sulfation, surface charge and surface charge density are measured with a variety of different methods, including elemental analysis (g S per g CNC), zeta-potential (mV) and conductometric titration (mmol sulfate ester groups per $\mathrm{kg} \mathrm{CNC}$ ), which often makes it difficult to make direct comparisons of data among studies.

In our study, elemental analysis showed that $\mathrm{S}$ content generally ranged between 0.5 and $2.0 \mathrm{mg} / \mathrm{g}$ for both CNCs and CSR, barring a couple of outliers such as run 7 with $6 \mathrm{mg}$ of S per $\mathrm{g} C \mathrm{NC}$ and run 19 with $3 \mathrm{mg}$ of S/g CSR (Table 6). ANOVA analysis was conducted excluding the outliers and only temperature was found to have a linear effect on the $\mathrm{S}$ content of CNCs (Additional file 1: Table S2). One possible explanation is that higher temperatures facilitated sulfonation of $\mathrm{CNCs}$ by reducing the amount of energy required to reach the activation energy of the esterification reaction. Previous studies have reported that $\mathrm{S}$ content or surface charge increased with increasing acid concentration (Chen et al. 2015) and hydrolysis duration (Kargarzadeh et al. 2012). Our study found a similar trend, but the two factors did not impact the $\mathrm{S}$ content significantly at $p<0.05$. In the cellulose-to$\mathrm{CNC}$ conversion process, the sulfation, formation and degradation of CNCs took place simultaneously, which resulted in $\mathrm{CNC}$ yield and S content being interrelated with respect to reaction severity (Chen et al. 2015; Wang et al. 2012). For the experimental ranges tested in this study, our results indicated that a threshold level of surface charge was imparted to the CNCs even at relatively milder conditions and did not change with a further increase in reaction severity. This indicated that $\mathrm{CNC}$ sulfation was not affected by reaction severity, in the same way as $\mathrm{CNC}$ formation or degradation was. Hence, we did not observe a strong correlation between acid concentration, hydrolysis duration and CNC S content.

Zeta-potential of CNC and CSR samples was measured using the DLS. Zeta-potential represents the potential difference that exists between particle surface and bulk liquid in an applied electric field (Reid et al. 2017), and provides significant insight into the colloidal stability of aqueous dispersions. Zeta-potential of CNCs generally ranged between -40 to $-60 \mathrm{mV}$ (Table 6), in agreement with previous reports (Kargarzadeh et al. 2012; Reid et al. 2017), and indicating very 
Table 6 Characterization of CNC and CSR using DLS, XRD and elemental analysis

\begin{tabular}{|c|c|c|c|c|c|c|c|}
\hline \multirow[t]{2}{*}{ Run no. } & \multirow[t]{2}{*}{ Particle size CNC (nm) } & \multicolumn{2}{|c|}{ Zeta-potential (mV) } & \multicolumn{2}{|c|}{$\mathrm{Crl}(\%)$} & \multicolumn{2}{|c|}{$S$ content $(\mathrm{mg} / \mathrm{g})$} \\
\hline & & CNC & CSR & $\mathrm{CNC}$ & CNF & CNC & CSR \\
\hline 1. & $167.9 \pm 2.9$ & $-(55.5 \pm 2.6)$ & & 75.8 & & $0.70 \pm 0.02$ & \\
\hline 2. & $136.2 \pm 2.2$ & $-(54.2 \pm 0.1)$ & & 82.9 & & $0.77 \pm 0.02$ & \\
\hline 3. & $165.5 \pm 0.3$ & $-(60.0 \pm 1.8)$ & $-(35.7 \pm 4.1)$ & 76.5 & 77.6 & $0.58 \pm 0.01$ & $0.51 \pm 0.01$ \\
\hline 4. & $216.1 \pm 3.0$ & $-(50.0 \pm 1.3)$ & $-(41.7 \pm 1.9)$ & 75.9 & 80.2 & $0.68 \pm 0.11$ & $0.51 \pm 0.01$ \\
\hline 5. & $184.9 \pm 1.2$ & $-(35.4 \pm 0.6)$ & $-(32.1 \pm 2.8)$ & 76.5 & 77.6 & $0.54 \pm 0.01$ & $0.50 \pm 0.02$ \\
\hline 6. & $172.6 \pm 4.8$ & $-(42.3 \pm 2.0)$ & & 76.9 & & $0.65 \pm 0.02$ & \\
\hline 7. & $196.8 \pm 3.1$ & $-(48.5 \pm 0.2)$ & $-(27.2 \pm 3.8)$ & 72.0 & 77.9 & $6.13 \pm 0.10$ & $0.69 \pm 0.03$ \\
\hline 8. & $169.9 \pm 6.6$ & $-(48.2 \pm 4.5)$ & & 75.1 & & $0.50 \pm 0.03$ & \\
\hline 9. & $214.4 \pm 3.4$ & $-(55.4 \pm 0.6)$ & - & 74.7 & 75.2 & $2.16 \pm 0.08$ & $0.37 \pm 0.02$ \\
\hline 10. & $137.1 \pm 3.9$ & $-(47.1 \pm 3.9)$ & & 77.0 & & $1.07 \pm 0.04$ & \\
\hline 11. & $200.9 \pm 10.4$ & $-(48.9 \pm 1.3)$ & $-(28.6 \pm 3.1)$ & 77.7 & 79.6 & $0.69 \pm 0.00$ & $0.46 \pm 0.02$ \\
\hline 12. & $209.9 \pm 3.7$ & $-(52.4 \pm 1.7)$ & - & 73.7 & 74.0 & $0.87 \pm 0.02$ & $0.40 \pm 0.01$ \\
\hline 13. & $136.5 \pm 1.9$ & $-(48.7 \pm 0.3)$ & & 75.1 & & $1.08 \pm 0.06$ & \\
\hline 14. & $212.0 \pm 6.7$ & $-(53.6 \pm 0.8)$ & $-(32.4 \pm 1.2)$ & 76.8 & 75.5 & $0.62 \pm 0.00$ & $0.37 \pm 0.00$ \\
\hline 15. & $143.0 \pm 2.1$ & $-(51.3 \pm 1.2)$ & & 75.7 & & $0.65 \pm 0.04$ & \\
\hline 16. & $153.6 \pm 1.5$ & $-(51.6 \pm 0.1)$ & & 75.9 & & $0.49 \pm 0.02$ & \\
\hline 17. & $193.9 \pm 3.6$ & $-(53.7 \pm 0.8)$ & - & 74.4 & 78.1 & $1.61 \pm 0.04$ & $0.26 \pm 0.01$ \\
\hline 18. & $143.6 \pm 2.6$ & $-(49.2 \pm 0.4)$ & & 76.1 & & $0.72 \pm 0.01$ & \\
\hline 19. & $159.2 \pm 1.6$ & $-(54.8 \pm 1.4)$ & $-(44.7 \pm 1.8)$ & 77.2 & 77.8 & $1.23 \pm 0.03$ & $2.99 \pm 0.09$ \\
\hline 20. & $148.4 \pm 0.5$ & $-(49.0 \pm 0.9)$ & & 81.2 & & $1.66 \pm 0.06$ & \\
\hline 21. & - & - & & - & & - & \\
\hline 22. & - & - & & - & & - & \\
\hline 23. & $133.0 \pm 2.5$ & $-(47.5 \pm 0.6)$ & & 75.3 & & $0.01 \pm 0.02$ & \\
\hline 24. & - & - & & - & & - & \\
\hline 25. & $149.3 \pm 1.0$ & $-(52.9 \pm 0.4)$ & & 78.8 & & $0.66 \pm 0.01$ & \\
\hline 26. & $150.5 \pm 3.4$ & $-(51.7 \pm 1.3)$ & & 76.9 & & $0.62 \pm 0.00$ & \\
\hline
\end{tabular}

*Zeta-potential of CSR from runs 9, 12 and 17 is not reported due to large fluctuations resulting from aggregation and sedimentation of large sized fibers

good colloidal stability of all samples. Attachment of anionic sulfate-ester groups to the surface of CNC particles resulted in electrostatic repulsion between particles and prevented aggregation. No settling of material was observed in any CNC sample (already separated from CSR) even months after their preparation, supporting the $\mathrm{S}$ content and zeta-potential data that were fairly consistent across all processing conditions. It was also evident from both $\mathrm{S}$ content and zeta-potential data of CSR that its degree of sulfation or surface charge was always lower than that of the corresponding $\mathrm{CNC}$ fraction (Table 6). However, it was sufficient to facilitate mechanical fibrillation into nanofibers, evident from the TEM image of CNFs obtained from run 17 (Fig. 4c), associated with the highest yield and lowest $\mathrm{S}$ content of CSR (Table 6).

\section{Particle size and dispersity}

A significant portion of behavior of CNCs, including self-assembly, phase separation and birefringence, rheological and mechanical properties imparted to nanocomposite materials, is attributed to its geometrical features, i.e., shape and size (Bai et al. 2009; Bondeson et al. 2006; Dong et al. 1998; Habibi et al. 2010; Reid et al. 2017; Sun et al. 2016). It is well-known that the morphology of CNCs is dependent on the raw material as well as the processing conditions used to prepare them. Longer hydrolysis duration and higher acid-to-pulp ratio have been shown to produce CNCs with shorter dimensions and narrower particle length distributions (BeckCandanedo et al. 2005; Bondeson et al. 2006; Chen et al. 2015; Dong et al. 1998; Kargarzadeh et al. 2012; Ren et al. 2014; Sun et al. 2016). Within the experimental ranges tested in our study, linear effects of acid concentration and temperature were found to have a significant effect on the hydrodynamic particle size of CNCs (Additional file 1: Table S3). Higher acid concentration and temperature resulted in shorter $\mathrm{CNCs}$, due to more acid molecules available per unit mass of cellulose and lower energy needed to reach the activation energy required for 
the esterification reaction, respectively. Average length ranged between 130 and $220 \mathrm{~nm}$ (Table 6), similar to what has been reported for $\mathrm{CNCs}$ extracted from woody biomass using strong acid hydrolysis (Beck-Candanedo et al. 2005; Chen et al. 2015; Sacui et al. 2014). Even though DLS does not provide absolute measurements of particle dimensions, it is a reliable tool for relative assessment of particle size and dispersity (Reid et al. 2017).

Dispersity $(\nexists)$ represents the breadth of particle size distribution within a sample; the numerical value of $Đ$ ranges from 0 (for a perfectly monodisperse sample with uniform particle size) to 1 (for a highly polydisperse sample with multiple particle size populations) (Danaei et al. 2018). In our study, dispersity of CNCs from pine kraft pulp ranged between 0.3 and 0.5 for all hydrolysis conditions tested, indicating moderately homogeneous particle size distribution. Previous studies have reported $Ð$ values approaching 0.2 for CNCs extracted from cotton (Fan and Li 2012; Sun et al. 2016), pointing towards a potential dependence of this parameter on the purity of raw material. Particle size and dispersity are considered important quality parameters for drug delivery applications (Danaei et al. 2018). Considering $\mathrm{CNCs}$ have demonstrated potential in such applications, production of $\mathrm{CNCs}$ with narrow size distributions is of key importance. Therefore, separation of CNCs from CSR using centrifugation is absolutely necessary to avoid having $\doteq$ values $>0.5$. Particle size of CSR is not reported in Table 6 because dispersity of these samples ranged between 0.7 and 1 . Size measurements of samples with $Ð \geq 0.7$ are not considered reliable (Danaei et al. 2018); however, high $Ð$ values were indicative of extremely broad particle size distributions for CSR. Although zetapotential data for some of the CSR samples (runs 9, 12 and 17) were unattainable, broad particle size distributions of CSR were concomitant with lower zeta-potential (Table 6).

\section{Conclusions}

In this study, strong acid hydrolysis parameters, including acid concentration, temperature, hydrolysis duration and pulp particle size, were optimized for extraction of CNCs from pre-extracted and kraft delignified loblolly pinewood. Acid concentration and temperature were found to have a significant effect on $\mathrm{CNC}$ yield. On the other hand, hydrolysis duration and particle size did not affect the yield significantly but could be fine-tuned to maximize cellulose-to-CNC conversion. While the optimal conditions obtained from the response surface methodology produced a mixture of CNCs and CSR, it is recommended not to separate the two streams at these conditions, but to implement conditions that favor complete hydrolysis of pulp to CNCs. Choices for maximizing the yields of either CNC or CSR streams (up to 60\% $\mathrm{w} / \mathrm{w}$ ) were provided, offering to manufacturers the use of identical processes for obtaining two different products. For harsher but high CNC yielding conditions, approximately $20 \%$ of holocellulose in the pulp degraded into simple sugars and oligomers. With better handling, CNC yields up to $70 \%$ were obtained by minimizing losses in processing steps such as washing. For milder but high CSR yielding conditions, holocellulose degradation was determined to be less than $5 \%$, where up to $15 \% \mathrm{CNCs}$ could be separately recovered. CSR had a very wide particle size distribution as well as lower surface charge, thus restricting its potential to be mixed with CNCs; however, its degree of sulfation was sufficient to facilitate conversion into CNFs through microfluidization. Since CNFs are typically produced using mechanical methods, future studies could investigate how those are different from sulfuric acid-produced CNFs. Overall, loblolly pine (softwood) kraft pulp was found to be a competitive source for CNC production; moreover, our study confirmed that it is absolutely necessary to use acid concentrations $<60 \%$ to maximize nanocellulose yield from wood pulp. In general, characteristic properties of CNCs, including crystallinity index, surface charge, particle size and dispersity, were found to be fairly uniform, implying consistency in quality within the extraction conditions tested. Lastly, even though the strong sulfuric acid process has been optimized for $\mathrm{CNC}$ production from a variety of cellulose-rich raw materials and is possibly the best approach, the process is still not environmentally friendly as it uses up to $58-60 \% \mathrm{w} / \mathrm{w}$ of acid. Such high concentrations of acid are associated with problems such as equipment corrosion, residual acid recycling and wastewater treatment. A few studies in the recent past have investigated "green" methods for CNC production; however, their yields were typically low. Sulfuric acid hydrolysis is possibly the best approach so far to obtain economically viable yields; however, future studies should address the issue of valorization of the waste stream, through development of efficient and scalable recycle and reuse systems for both acid and water, in order to make the process more sustainable.

\section{Supplementary information}

Supplementary information accompanies this paper at https://doi. org/10.1186/s40643-020-00302-0.

Additional file 1. Additional figures and tables.

\section{Abbreviations}

CNC: Cellulose nanocrystal; CNF: Cellulose nanofiber; CCD: Central composite design; RSM: Response surface methodology; CSR: Cellulosic solid residue; DOE: Design of experiments; ECF: Elemental chlorine free; HPLC: High performance liquid chromatography; RPM: Revolutions per minute; TEM: Transmission electron microscope; XRD: X-ray diffraction; $\mathrm{Crl}$ : Crystallinity index; ICP-OES: Inductively coupled plasma optical emission spectrometry; DLS: 
Dynamic light scattering; MCC: Microcrystalline cellulose; ANOVA: Analysis of variance; RMSE: Root mean square error.

\section{Acknowledgements}

The authors would like to thank A. Kuchuk and E. Martin at the Institute for Nanoscience and Engineering, University of Arkansas, Fayetteville, for their assistance with XRD analysis and TEM imaging, respectively. The authors would also like to thank C. Hamilton from the Center for Renewable Carbon, University of Tennessee, Knoxville for conducting the ICP-OES analysis.

\section{Authors' contributions}

J-WK and GK conceived and designed the experiments. GK, AD and KR performed experiments. All authors discussed the results. GK, KR, DJC and J-WK co-wrote the paper. All authors read and approved the final manuscript.

\section{Funding}

This project was supported by the Center for Advanced Surface Engineering (CASE) under the National Science Foundation (NSF) grant number OIA1457888 and the Arkansas EPSCoR program, ASSET III.

\section{Availability of data and materials}

All data supporting this article's conclusion are available.

\section{Ethics approval and consent to participate}

Not applicable.

\section{Consent for publication}

Not applicable.

\section{Competing interests}

The authors declare that they have no competing interests.

\section{Author details}

${ }^{1}$ Department of Biological and Agricultural Engineering, University of Arkansas, Fayetteville, AR 72701, USA. ${ }^{2}$ Institute for Nanoscience and Engineering, University of Arkansas, Fayetteville, AR 72701, USA. ${ }^{3}$ Department of Biosystems Engineering and Soil Science, University of Tennessee Institute of Agriculture, Knoxville, TN 37996, USA. ${ }^{4}$ Center for Renewable Carbon, University of Tennessee Institute of Agriculture, Knoxville, TN 37996, USA. ${ }^{5}$ Department of Chemistry and Biochemistry, University of Arkansas, Fayetteville, AR 72701 USA.

Received: 5 November 2019 Accepted: 10 March 2020

Published online: 31 March 2020

\section{References}

Ahvenainen P, Kontro I, Svedström K (2016) Comparison of sample crystallinity determination methods by $\mathrm{X}$-ray diffraction for challenging cellulose I materials. Cellulose 23:1073-1086. https://doi.org/10.1007/s1057 0-016-0881-6

Amin KNM, Annamalai PK, Morrow IC, Martin D (2015) Production of cellulose nanocrystals via a scalable mechanical method. RSC Adv 5:57133-57140. https://doi.org/10.1039/C5RA06862B

Araki J, Wada M, Kuga S, Okano T (1998) Flow properties of microcrystalline cellulose suspension prepared by acid treatment of native cellulose. Colloid Surf A 142:75-82. https://doi.org/10.1016/S0927-7757(98)00404-X

Area MC, Popa VI (2014) Anatomy, structure and chemistry of fibrous materials. In: Area MC, Popa VI (eds) Wood fibres for papermaking. Smithers Rapra Technology, New York, pp 29-40

Bai W, Holbery J, Li K (2009) A technique for production of nanocrystalline cellulose with a narrow size distribution. Cellulose 16:455-465. https://doi. org/10.1007/s10570-009-9277-1

Battista OA (1950) Hydrolysis and crystallization of cellulose. Ind Eng Chem 42(3):502-507. https://doi.org/10.1021/ie50483a029

Beck-Candanedo S, Roman M, Gray DG (2005) Effect of reaction conditions on the properties and behavior of wood cellulose nanocrystal suspensions. Biomacromol 6:1048-1054.
Behera S, Arora R, Nandhagopal N, Kumar S (2014) Importance of chemical pretreatment for bioconversion of lignocellulosic biomass. Renew Sustain Energy Rev 36:91-106. https://doi.org/10.1016/j.rser.2014.04.047

Benini KCCC, Voorwald HJC, Cioffi MOH, Rezende MC, Arantes V (2018) Preparation of nanocellulose from Imperata brasiliensis grass using Taguchi method. Carbohydr Polym 192:337-346. https://doi.org/10.1016/j.carbp ol.2018.03.055

Bezerra MA, Santelli RE, Oliveira EP, Villar LS, Escaleira LA (2008) Response surface methodology (RSM) as a tool for optimization in analytical chemistry. Talanta 76:965-977. https://doi.org/10.1016/j.talanta.2008.05.019

Biermann CJ (1996) Handbook of pulping and papermaking, 2nd edn. Academic Press, Cambridge

Bondeson D, Mathew A, Oksman K (2006) Optimization of the isolation of nanocrystals from microcrystalline cellulose by acid hydrolysis. Cellulose 13:171-180. https://doi.org/10.1007/s10570-006-9061-4

Börjesson M, Westman G (2015) Crystalline nanocellulose-preparation, modification and properties. In: Poletto M (ed) Cellulose-fundamental aspects and current trends. IntechOpen, London. https://doi. org/10.5772/61899

Bragg DC (2011) Forests and forestry in Arkansas during the last two centuries. In: Riley LE, Haase DL, Pinto JR (tech coords) National proceedings: forest and conservation nursery associations, 2010. In: Proceedings of RMRSP-65. Fort Collins, CO: U.S. Department of Agriculture, Forest Service, Rocky Mountain Research Station, p. 3-9

Brinchi L, Cotana F, Fortunati E, Kenny JM (2013) Production of nanocrystalline cellulose from lignocellulosic biomass: technology and applications. Carbohydr Polym 94:154-169. https://doi.org/10.1016/j.carbpol.2013.01.033

Chen L, Wang Q, Hirth K, Baez C, Agarwal UP, Zhu JY (2015) Tailoring the yield and characteristics of wood cellulose nanocrystals (CNC) using concentrated acid hydrolysis. Cellulose 22:1753-1762. https://doi.org/10.1007/ s10570-015-0615-1

Chen L, Zhu JY, Baez C, Kitin P, Elder T (2016) Highly thermal-stable and functional cellulose nanocrystals and nanofibrils produced using fully recyclable organic acids. Green Chem 18:3835-3843. https://doi.org/10.1039/ C6GC00687F

Danaei M et al (2018) Impact of particle size and polydispersity index on the clinical applications of lipidic nanocarrier systems. Pharmaceutics 10:57. https://doi.org/10.3390/pharmaceutics10020057

Dean A, Voss D, Draguljić D (2017) Response surface methodology. In: Dean A, Voss D, Draguljić D (eds) Design and analysis of experiments. Springer, Cham, pp 565-614. https://doi.org/10.1007/978-3-319-52250-0_16

Dong XM, Revol JF, Gray DG (1998) Effect of microcrystallite preparation conditions on the formation of colloid crystals of cellulose. Cellulose 5:19-32. https://doi.org/10.1023/A:1009260511939

Dong S, Bortner MJ, Roman M (2016) Analysis of the sulfuric acid hydrolysis of wood pulp for cellulose nanocrystal production: a central composite design study. Ind Crop Prod 93:76-87. https://doi.org/10.1016/j.inder op.2016.01.048

Dufresne A (2013) Nanocellulose: a new ageless bionanomaterial. Mater Today 16:220-227. https://doi.org/10.1016/j.mattod.2013.06.004

Fan JS, Li YH (2012) Maximizing the yield of nanocrystalline cellulose from cotton pulp fiber. Carbohydr Polym 88:1184-1188. https://doi.org/10.1016/j. carbpol.2012.01.081

George J, Sabapathi SN (2015) Cellulose nanocrystals: synthesis, functional properties, and applications. Nanotechnol Sci Appl 8:45-54. https://doi. org/10.2147/NSA.S64386

Habibi Y, Lucia LA, Rojas OJ (2010) Cellulose nanocrystals: chemistry, selfassembly, and applications. Chem Rev 110:3479-3500. https://doi. org/10.1021/cr900339w

Hamad WY, Hu TQ (2010) Structure-process-yield interrelations in nanocrystalline cellulose extraction. Can J Chem Eng 88:392-402. https://doi. org/10.1002/cjce.20298

Hamer G (2003) Solid waste treatment and disposal: effects on public health and environmental safety. Biotechnol Adv 22:71-79. https://doi. org/10.1016/j.biotechadv.2003.08.007

Houtman C (2018) Lessons learned from 150 years of pulping wood. In: Beckham GT (ed) Energy and environment series no. 19: lignin valorization: emerging approaches. Royal Society of Chemistry, Cambridge, pp 62-74

Hult EL, Iversen T, Sugiyama J (2003) Characterization of the supermolecular structure of cellulose in wood pulp fibres. Cellulose 10:103-110. https:// doi.org/10.1023/A:1024080700873 
Jonoobi M, Oladi R, Davoudpour Y, Oksman K, Dufresne A, Hamzeh Y, Davoodi $R$ (2015) Different preparation methods and properties of nanostructured cellulose from various natural resources and residues: a review. Cellulose 22:935-969. https://doi.org/10.1007/s10570-015-0551-0

Kargarzadeh H, Ahmad I, Abdullah I, Dufresne A, Zainudin SY, Sheltami RM (2012) Effects of hydrolysis conditions on the morphology, crystallinity, and thermal stability of cellulose nanocrystals extracted from kenaf bast fibers. Cellulose 19:855-866. https://doi.org/10.1007/s10570-012-9684-6

Lacerda TM, Zambon MD, Frollini E (2013) Effect of acid concentration and pulp properties on hydrolysis reactions of mercerized sisal. Carbohydr Polym 93:347-356. https://doi.org/10.1016/j.carbpol.2012.10.039

Lee HV, Hamid SBA, Zain SK (2014) Conversion of lignocellulosic biomass to nanocellulose: structure and chemical process. Sci World J 2014:1-20. https://doi.org/10.1155/2014/631013

Li Y, Liu Y, Chen W, Wang Q, Liu Y, Li J, Yu H (2016) Facile extraction of cellulose nanocrystals from wood using ethanol and peroxide solvothermal pretreatment followed by ultrasonic nanofibrillation. Green Chem 18:1010-1018. https://doi.org/10.1039/C5GC02576A

Lin N, Dufresne A (2014) Surface chemistry, morphological analysis and properties of cellulose nanocrystals with gradiented sulfation degrees. Nanoscale 6:5384-5393. https://doi.org/10.1039/C3NR06761K

Lu Z, Fan L, Zheng H, Lu Q, Liao Y, Huang B (2013) Preparation, characterization and optimization of nanocellulose whiskers by simultaneously ultrasonic wave and microwave assisted. Bioresour Technol 146:82-88. https://doi. org/10.1016/j.biortech.2013.07.047

Mishra RK, Sabu A, Tiwari SK (2018) Materials chemistry and the futurist ecofriendly applications of nanocellulose: status and prospect. I Saudi Chem Soc 22:949-978. https://doi.org/10.1016/j.jscs.2018.02.005

Moon RJ, Martini A, Nairn J, Simonsen J, Youngblood J (2011) Cellulose nanomaterials review: structure, properties and nanocomposites. Chem Soc Rev 40:3941-3994. https://doi.org/10.1039/C0CS00108B

Mukherjee SM, Woods HJ (1953) X-ray and electron microscope studies of the degradation of cellulose by sulphuric acid. BBA 10:499-511. https://doi. org/10.1016/0006-3002(53)90295-9

Nam S, French AD, Condon BD, Concha M (2016) Segal crystallinity index revisited by the simulation of $X$-ray diffraction patterns of cotton cellulose I $\beta$ and cellulose II. Carbohydr Polym 135:1-9. https://doi.org/10.1016/j. carbpol.2015.08.035

Ngwabebhoh FA, Erdem A, Yildiz U (2018) A design optimization study on synthesized nanocrystalline cellulose, evaluation and surface modification as a potential biomaterial for prospective biomedical applications. Int J Biol Macromol 114:536-546. https://doi.org/10.1016/j.ijbiomac.2018.03.155

Novo LP, Bras J, Garcia A, Belgacem N, Curvelo AAS (2015) Subcritical water: a method for green production of cellulose nanocrystals. ACS Sust Chem Eng 3:2839-2846. https://doi.org/10.1021/acssuschemeng.5b00762

Park S, Baker JO, Himmel ME, Parilla PA, Johnson DK (2010) Cellulose crystallinity index: measurement techniques and their impact on interpreting cellulase performance. Biotechnol Biofuels 3:10. https://doi. org/10.1186/1754-6834-3-10

Peng BL, Dhar N, Liu HL, Tam KC (2011) Chemistry and applications of nanocrystalline cellulose and its derivatives: a nanotechnology perspec tive. Can J Chem Eng 89:1 191-1206. https://doi.org/10.1002/cjce.20554

Pettersen RC (1984) The chemical composition of wood. In: Rowell R (ed) The chemistry of solid wood, vol 207. American Chemical Society, Washington, pp 57-126. https://doi.org/10.1021/ba-1984-0207.ch002

Poletto M (2015) Cellulose-fundamental aspects and current trends. IntechOpen. https://doi.org/10.5772/59889

Poletto M, Pistor V, Zeni M, Zattera AJ (2011) Crystalline properties and decomposition kinetics of cellulose fibers in wood pulp obtained by two pulping processes. Polym Degrad Stabil 96:679-685. https://doi.org/10.1016/j. polymdegradstab.2010.12.007
Postek MT, Moon RJ, Rudie AW, Bilodeau MA (2013) Production and applications of cellulose nanomaterials. TAPPI Press, Atlanta

Rajan K, Djioleu A, Kandhola G, Labbé N, Sakon J, Carrier DJ, Kim J-W (2020) Investigating the effects of hemicellulose pre-extraction on the production and characterization of loblolly pine nanocellulose. Cellulose (accepted). https://doi.org/10.1007/s10570-020-03018-8

Reid MS, Villalobos M, Cranston ED (2017) Benchmarking cellulose nanocrystals: from the laboratory to industrial production. Langmuir 33:15831598. https://doi.org/10.1021/acs.langmuir.6b03765

Ren S, Sun X, Lei T, Wu Q (2014) The effect of chemical and high-pressure homogenization treatment conditions on the morphology of cellulose nanoparticles. J Nanomater 2014:582913. https://doi. org/10.1155/2014/582913

Sacui IA et al (2014) Comparison of the properties of cellulose nanocrystals and cellulose nanofibrils isolated from bacteria, tunicate, and wood processed using acid, enzymatic, mechanical, and oxidative methods. ACS Appl Mater Inter 6:6127-6138. https://doi.org/10.1021/am500359f

Segal L, Creely JJ, Martin AE Jr, Conrad CM (1959) An empirical method for estimating the degree of crystallinity of native cellulose using the X-ray diffractometer. Text Res J 29:786-794.

Selim IZ, Zikry AAF, Gaber SH (2005) Physicochemical properties of prepared cellulose sulfates: II from linen pulp bleached by the $\mathrm{H}_{2} \mathrm{O}_{2}$ method. Polymer Plast Technol Eng 43(5):1387-1402. https://doi.org/10.1081/ PPT-200030194

Seo Y-R, Kim J-W, Hoon S, Kim J, Chung JH, Lim KT (2018) Cellulose-based nanocrystals: sources and applications via agricultural byproducts. J Biosyst Eng 43(1):59-71. https://doi.org/10.5307/JBE.2018.43.1.059

Shatkin JA, Wegner TH, Bilek EM, Cowie J (2014) Market projections of cellulose nanomaterial-enabled products—part 1: applications. Tappi J 13:9-16

Sinha A, Martin EM, Lim KT, Carrier DJ, Han H, Zharov VP, Kim J-W (2015) Cellulose nanocrystals as advanced "green" materials for biological and biomedical engineering. J Biosyst Eng 40:373-393. https://doi.org/10.5307/ JBE.2015.40.4.373

Sun B, Zhang M, Hou Q, Liu R, Wu T, Si C (2016) Further characterization of cellulose nanocrystal (CNC) preparation from sulfuric acid hydrolysis of cotton fibers. Cellulose 23:439-450. https://doi.org/10.1007/s1057 $0-015-0803-z$

Thygesen A, Oddershede J, Lilholt H, Thomsen AB, Stahl K (2005) On the determination of crystallinity and cellulose content in plant fibres. Cellulose 12:563-576. https://doi.org/10.1007/s10570-005-9001-8

Wang QQ, Zhu JY, Reiner RS, Verrill SP, Baxa U, McNeil SE (2012) Approaching zero cellulose loss in cellulose nanocrystal (CNC) production: recovery and characterization of cellulosic solid residues (CSR) and CNC. Cellulose 19:2033-2047. https://doi.org/10.1007/s10570-012-9765-6

Wang Q, Zhao X, Zhu JY (2014) Kinetics of strong acid hydrolysis of a bleached kraft pulp for producing cellulose nanocrystals (CNCs). Ind Eng Chem Res 53:11007-11014. https://doi.org/10.1021/ie501672m

Zhang R, Liu Y (2018) High energy oxidation and organosolv solubilization for high yield isolation of cellulose nanocrystals (CNC) from Eucalyptus hardwood. Sci Rep 8:1-11. https://doi.org/10.1038/s41598-018-34667-2

\section{Publisher's Note}

Springer Nature remains neutral with regard to jurisdictional claims in published maps and institutional affiliations. 